\title{
Diameter-dependent thermoelectric figure of merit in single-crystalline Bi nanowires
}

\section{Citation}

Kim, Jeongmin, Seunghyun Lee, Yuri M. Brovman, Philip Kim, and Wooyoung Lee. 2015. "Diameter-Dependent Thermoelectric Figure of Merit in Single-Crystalline Bi Nanowires." Nanoscale 7 (11): 5053-5059. doi:10.1039/c4nr06412g.

\section{Published Version}

doi:10.1039/C4NR06412G

\section{Permanent link}

http://nrs.harvard.edu/urn-3:HUL.InstRepos:27715678

\section{Terms of Use}

This article was downloaded from Harvard University's DASH repository, and is made available under the terms and conditions applicable to Other Posted Material, as set forth at http:// nrs.harvard.edu/urn-3:HUL.InstRepos:dash.current.terms-of-use\#LAA

\section{Share Your Story}

The Harvard community has made this article openly available.

Please share how this access benefits you. Submit a story.

\section{Accessibility}




\title{
Nanoscale
}

\section{PAPER}

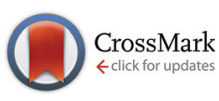

Cite this: Nanoscale, 2015, 7, 5053

\section{Diameter-dependent thermoelectric figure of merit in single-crystalline Bi nanowires $\uparrow$}

\author{
Jeongmin Kim, ${ }^{a}$ Seunghyun Lee, ${ }^{a}$ Yuri M. Brovman, ${ }^{b}$ Philip Kim ${ }^{b}$ and \\ Wooyoung Lee*a
}

\begin{abstract}
The diameter-dependent thermoelectric properties of individual single-crystalline Bi nanowires grown by the on-film formation of nanowires method have been investigated. The electrical resistivity, Seebeck coefficient, and thermal conductivity were measured as functions of the nanowire diameter using an individual nanowire device. The thermoelectric figure of merit $(Z T)$ calculated from the measured thermoelectric properties shows an increase from the bulk value to a maximum value of 0.28 at $109 \mathrm{~nm}$ diameter, followed by a decrease upon further decreasing the diameter. This non-monotonic diameter dependence of $Z T$ in $\mathrm{Bi}$ nanowires reveals simultaneous positive and negative contributions to the thermoelectric efficiency, driven by the change in intrinsic properties, which originates from the diameter-dependent classical and quantum size effects.
\end{abstract}

Received 31st October 2014

Accepted 9th January 2015

DOI: $10.1039 / c 4 n r 06412 \mathrm{~g}$

www.rsc.org/nanoscale way to achieve a high Seebeck coefficient using Sn-doped Bi NW. ${ }^{10}$ They confirmed the enhancement of the Seebeck coefficient by the Sn doping in relation to that of pure Bi NWs,${ }^{11}$ but the enhanced values could not exceed those in bulk $\mathrm{Bi}$ (2001-2002). Alternatively, Huber et al. fabricated arraytype $^{12-14}$ and individual ${ }^{15,16}$ single-crystalline Bi NWs using the AAO templates and the Ulitovsky method, respectively. These NWs with diameters less than $50 \mathrm{~nm}$ displayed transport properties, which were predominantly governed by surface states, including electrical conductivity and the Seebeck coefficient, in quantum oscillations such as Shubnikov-de Haas $(\mathrm{SdH})^{12,13}$ and Aharonov-Bohm ${ }^{16}$ oscillations. However, the maximum measured Seebeck coefficient ${ }^{14,15}$ was similar to the value in bulk Bi (2004-2011). Moreover, the fourprobe technique used to measure the absolute properties of the NWs could not be applied owing to the limitation of the growth methods, the array structure of the AAO template, or the glass tube used in the Ulitovsky method. Recently, Shapira et al. reported the enhancement of the Seebeck coefficient using a one-dimensional $\mathrm{Bi} \mathrm{NW}$, exploiting the reduction of the diameter caused by the electromigration (EM) effect (2012). ${ }^{17}$ However, because this reduction of the diameter using EM was difficult to determine, the resistivity of the NWs was not obtained. Furthermore, the absence of thermal conductivity measured in the above NWs suggests that the dimensionless thermoelectric figure of merit $(Z T)$, which is used to estimate the efficiency of a thermoelectric material, has never been evaluated in Bi NWs.

To evaluate $Z T$ in Bi NWs, we first successfully fabricated single-crystalline Bi NWs using a novel growth method the onfilm formation of nanowires (OFFON), in 2009. ${ }^{18}$ As shown in

\footnotetext{
${ }^{a}$ Department of Materials Science and Engineering Yonsei University, 134 Shinchon, Seoul 120-749, Korea.E-mail: wooyoung@yonsei.ac.kr

${ }^{b}$ Department of Physics, Columbia University, New York, New York 10027, USA

$\dagger$ Electronic supplementary information (ESI) available. See DOI: 10.1039/ c4nr06412g
} 

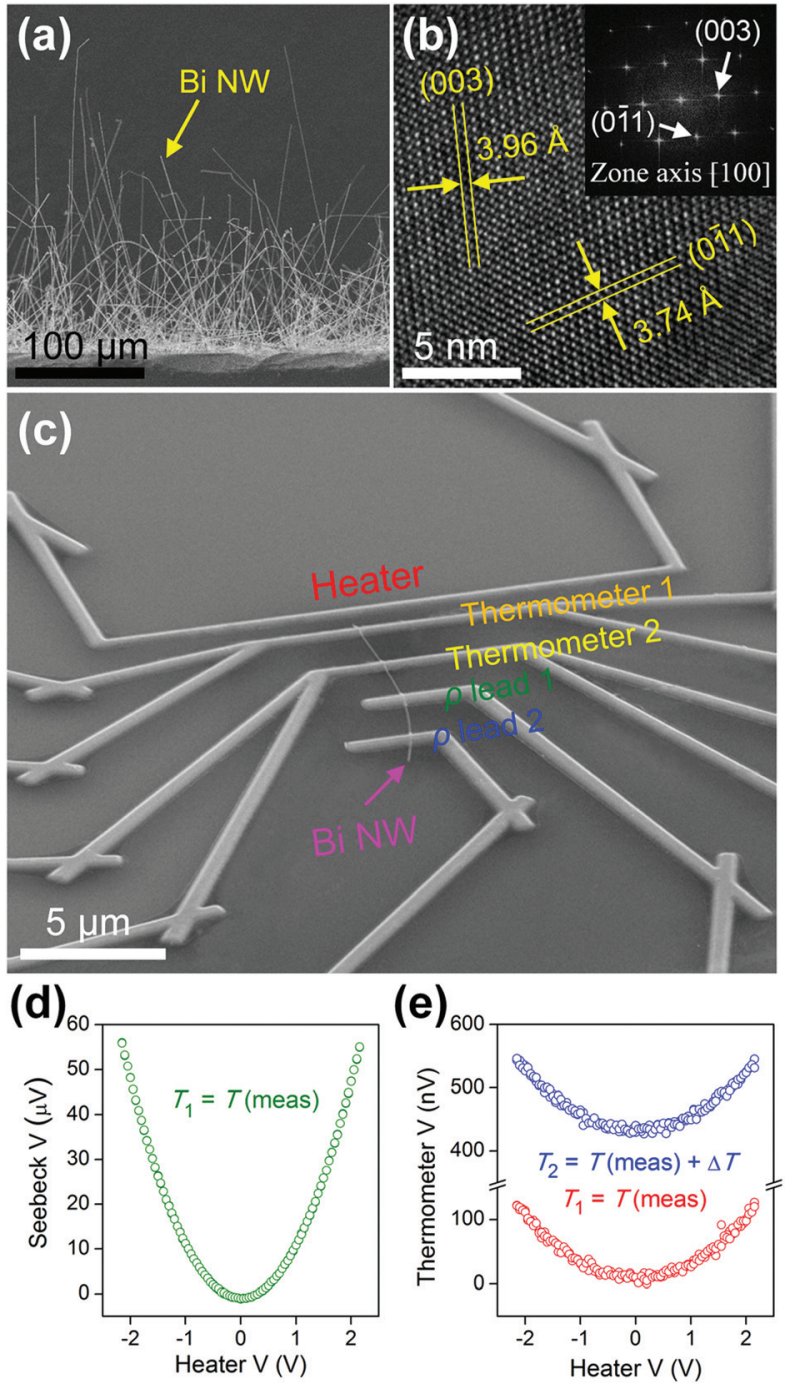

Fig. 1 Single-crystalline Bi NWs grown by OFFON and a TE device for the measurement of electrical thermoelectric properties: (a) SEM image of as-grown Bi NWs on a Bi thin film. (b) HRTEM image of a typical Bi NW. The sample was prepared by slicing normal to the growth direction, and thus the [100] zone axis of the SAED pattern (inset) indicates the growth direction of the Bi NW. (c) SEM image of a TE device based on an individual Bi NW. The clear parabolic signals of (d) the Seebeck and (e) the thermometer voltages as functions of the heater voltage confirm the reliability of the measurements.

Fig. 1a, the high aspect ratio of Bi NWs and the absence of supporting parts (such as the template and tube) allow the use of various measurement techniques such as the four-probe device $^{19-22}$ and the suspended microdevice. ${ }^{23}$ These preliminary studies investigated the quantum transport phenomena and the thermal conductivity anisotropy, and confirmed the high quality of the single-crystalline properties. ${ }^{19-23}$ In this study, we present the diameter-dependent thermoelectric properties of single-crystalline Bi NWs grown by OFFON. The electrical resistivity $(\rho)$ and Seebeck coefficient $(S)$ of the Bi NWs were measured as the functions of diameter $(d)$ and temperature $(T)$ using thermoelectric (TE) devices based on a single
NW. The diameter dependence of the thermoelectric power factor ( $\mathrm{PF}, S^{2} / \rho$ ) was calculated using the measured resistivity and the Seebeck coefficient. The diameter-dependent thermal conductivity $(\kappa)$ of the Bi NWs was obtained using suspended microdevices based on a single NW. The dimensionless thermoelectric figure of merit $\left(Z T=S^{2} T / \rho \kappa\right)$ was also calculated as a function of diameter. Its non-monotonic behavior, observed as the diameter decreases, indicates that the change in the intrinsic properties originating from QSEs affects the thermoelectric properties both positively and negatively. This interpretation, which takes into account the SMSC transition in low-dimensional Bi, also accounts for the smaller Seebeck coefficient of Bi NWs than that of bulk Bi, reported in previous studies. ${ }^{9,11,14,15}$

\section{Experimental section}

\section{Materials}

High-quality single-crystalline $\mathrm{Bi}$ NWs were obtained by OFFON. ${ }^{18-23}$ The NWs were grown spontaneously from Bi thin films to release the stress induced by the heat treatment without the supporting parts or catalysts. The Bi films, less than $50 \mathrm{~nm}$ thick, were deposited onto thermally oxidized $\mathrm{Si}$ (100) substrates at a rate of $44 \AA \mathrm{s}^{-1}$ using a custom-made ultrahigh vacuum (UHV) sputtering system with an Ar gas pressure of 2 mTorr. To reduce the NW diameter, the substrate was cooled with liquid nitrogen during the sputtering process; moreover, the diameter was found to be proportional to the grain size of the thin film. ${ }^{24}$ The heat treatment was performed in a custom-made UHV furnace at $250{ }^{\circ} \mathrm{C}$ for 5 to 10 hours. The annealing time controls the NW length. Fig. 1b shows a high-resolution transmission electron microscopy (HRTEM, FE-TEM JEM-2100F JEOL) image and a selected-area electron diffraction (SAED) pattern (inset) of a $\mathrm{Bi}$ NW prepared by slicing perpendicular to the NW direction using a focused ion beam (FIB, Quanta 3D FEG FEI).

\section{Device fabrication}

The TE devices, based on a single Bi NW for the measurement of the electrical thermoelectric properties ( $\rho$ and $S$ ), were successfully fabricated using electron-beam lithography (JSM-7001F JEOL and ELPHY Quantum Raith) and Ar plasma etching (custom-made system) (Fig. 1c). ${ }^{18-22}$ The as-grown Bi NWs were dispersed by direct contact with a thermally oxidized Si (100) substrate, on which alignment markers were patterned by photolithography. The position and orientation of a selected NW were defined by the digitalization of the coordinates from a scanning electron microscopy (SEM) image for the electron-beam lithography. To remove the native oxide layer of the Bi NWs, Ar plasma etching was performed before $\mathrm{Cr}(5 \mathrm{~nm}) / \mathrm{Au}(100-300 \mathrm{~nm})$ metallization for electrodes, using a UHV DC sputtering system (custom-made).

Suspended microdevices, each based on a single Bi NW, served to measure the thermal conductivity and were fabricated using a drop-casting method and by the deposition of a 
thermal contact. ${ }^{23}$ The as-grown Bi NWs on the Bi thin film/Si substrate were rinsed in isopropyl alcohol before drop-casting onto the suspended microdevices. To improve the thermal contact between the $\mathrm{Bi} \mathrm{NW}$ and the microdevice, a Pt/C composite was deposited onto the contact region by FIB. ${ }^{23,25,26}$ The second deposition and measurement were performed after the first measurement to allow the subtraction of the thermalcontact dependence.

\section{Measurement technique}

As shown in Fig. 1c, the electrical thermoelectric properties $(\rho$ and $S$ ) of the Bi NW were measured using a micro-heater, two thermometers, and two resistivity probes. The four different electrodes (except the micro-heater) were used for the four-probe measurement, which yields an absolute value for the NW resistance independent of the contact resistance. The resistivity was calculated by the relation, $\rho=R \pi(d / 2)^{2} / L$, where $R$ and $L$ are the resistance and channel length, respectively. To estimate the error in $\rho$, we considered two different channel lengths to obtain upper and lower limits: edge-to-edge $\left(\rho_{\max }\right)$ and center-to-center $\left(\rho_{\min }\right)$. Repeated measurements showed negligible variation.

Compared to the resistivity, measuring the Seebeck coefficient is complicated. ${ }^{27-29}$ To produce a Seebeck voltage, a temperature gradient was generated by Joule heating using the micro-heater. As shown in Fig. 1d, the Seebeck voltage between the two thermometer probes was proportional to the square of the heater voltage applied to the micro-heater, following the heating power $P=V^{2} / R$. The temperature difference was measured using the resistance increase of each thermometer, which also shows a quadratic relationship with the heater voltage (Fig. 1e). The resistance of the thermometers was measured as a voltage signal, which was obtained by a four-probe method. To calibrate the temperature-voltage ratio for each thermometer, the measurement was performed at two different ambient temperatures (See Fig. S1 in the ESI $\dagger$ ). Finally, the Seebeck coefficient was calculated by dividing the Seebeck voltage by the temperature difference between the thermometers. The reliability of this method was confirmed by the clear parabolas shown by the Seebeck and thermometer voltages, and the errors were obtained from the repeated sweeps of the heater voltage. The precise measurements of the Seebeck and thermometer voltages were done with a nano-voltmeter (2182A KEITHLEY) and a lock-in amplifier (SR850 Stanford Research Systems), respectively.

The suspended microdevice for the thermal conductivity measurement consisted of two suspended silicon nitride $\left(\mathrm{SiN}_{x}\right)$ membranes, each supported by five $\mathrm{SiN}_{x}$ beams. One Pt electrode and a Pt resistance thermometer (PRT) coil with four-probe electrodes were patterned on each membrane. The PRT coil served simultaneously as a micro-heater and a thermometer. Joule heating by the PRT coil on the heating membrane was transmitted to the sensing membrane through a Bi NW. Using the four-probe electrodes, the resistance of each membrane was measured using a lock-in amplifier and converted to a temperature in the same manner as for the
Seebeck coefficient measurement. The thermal conductance of the Bi NW was calculated as $G=\Delta T_{\mathrm{s}} P /\left(\Delta T_{\mathrm{h}}{ }^{2}-\Delta T_{\mathrm{s}}{ }^{2}\right)$, where $T_{\mathrm{h}}$, and $T_{\mathrm{s}}$ are the temperatures of the heating and sensing membranes, respectively. ${ }^{23}$ Finally, the thermal conductivity was obtained using the NW length $(L)$ and cross-sectional area $\left(\pi(d / 2)^{2}\right)$ as $\kappa=G \pi(d / 2)^{2} / L$. Errors were estimated from five repeated sweeps of the heater voltage. All measurements, including those for the resistivity and Seebeck coefficient, were performed in an evacuated cryostat (custom-made, $\sim 5.0 \times 10^{-6}$ Torr) to eliminate convection heat losses. A detailed description of the measurements is given elsewhere. ${ }^{21,23,27-29}$

\section{Results and discussion}

\section{Single-crystalline Bi nanowires}

The HRTEM image and SAED pattern confirm that the OFFON-grown Bi NW is a high-quality single-crystal in the [100] direction (inset of Fig. 1b). As shown in Fig. 1b, the lattice-fringe spacings are 3.96 and $3.74 \AA$, which are consistent with the lattice plane spacings of (003) and (0-11) in $\mathrm{Bi}$, respectively ( $a=4.546 \AA$ and $c=11.862$, JCPDS card no. 851329). ${ }^{30}$ The large $\mathrm{MR}$ and definite $\mathrm{SdH}$ oscillations seen in previous studies also indicates the high-quality of the singlecrystalline OFFON-grown Bi NWs. ${ }^{18-20,22}$ Twelve different Bi NWs with diameters ranging from 21 to $506 \mathrm{~nm}$ were used for the TE devices and additional four-probe devices were fabricated to measure the diameter dependence of the resistivity.

\section{Electrical resistivity}

Fig. 2a shows a significant variation in the temperature dependence of the NW resistance with diameters 21, 27, 40, 59, 67, 109, and $178 \mathrm{~nm}$. In general, the resistance (resistivity) of metals decreases with decreasing temperature, owing to the increase in the mobility, which is caused by the reduction of carrier-acoustic phonon scattering. Conversely, in typical semiconductors, a reduction in carrier concentration leads to a decrease in resistance as the temperature decreases. However, In the case of the semimetal $\mathrm{Bi}$, the metallic and semiconducting characteristics coexist because of the small carrier concentration of $\mathrm{Bi}\left(2.7 \times 10^{17}-3.0 \times 10^{18} \mathrm{~cm}^{-3}\right.$ at $\left.2-300 \mathrm{~K}\right) .^{5,7,12,15}$ The temperature dependence of the resistance is determined by the competition between the mobility and the carrier concen-

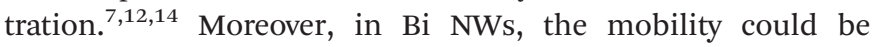
varied not only by the carrier scattering from the non-specular surface but also by the strong coupling effect in the L-point sub-bands. ${ }^{2,31}$ Bi NWs with a large diameter $(d \gg 100 \mathrm{~nm})$, show bulk-like metallic behavior, whereas, in the intermediate diameter range of $40<d<110 \mathrm{~nm}$, the mobility contribution diminishes with decreasing diameter because of the spatial constraints of the Bi NWs. Furthermore, the reduced carrier concentration due to $\mathrm{QSE}^{22}$ results in the semiconducting behavior of the Bi NWs. For small diameters $(d<30 \mathrm{~nm})$, however, the temperature dependence of resistance returned to metallic. In Bi NWs ${ }^{12-14,16}$ and Bi thin films ${ }^{31,32}$ with dimensions below $50 \mathrm{~nm}$, the metallic surface-state effects reportedly 


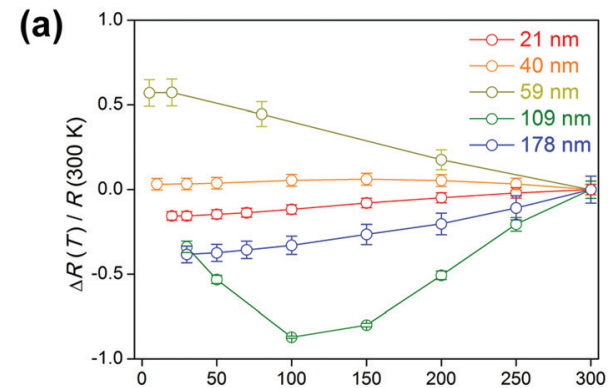

(c)

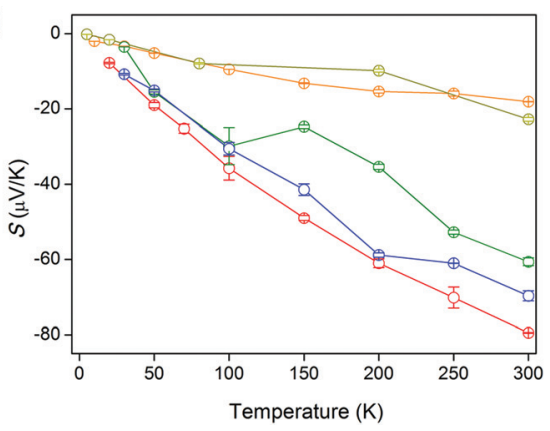

(b)

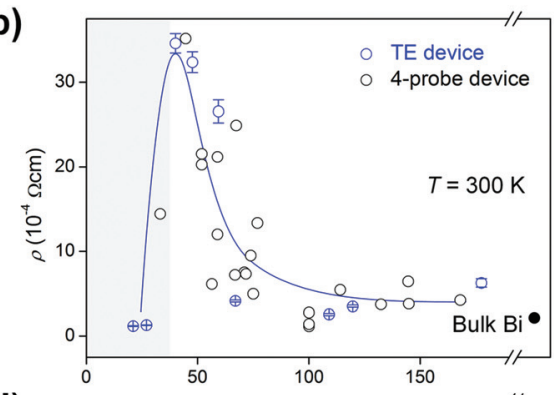

(d)

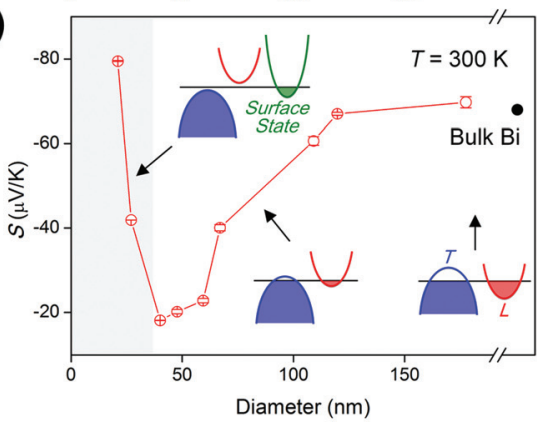

Fig. 2 Diameter- and temperature-dependent electrical thermoelectric properties of single-crystalline Bi NWs: Resistivity (normalized resistance) was measured as a function of (a) temperature and (b) diameter. The Seebeck coefficient is also plotted against (c) temperature and (d) diameter. The diameter dependence of both the resistivity and the Seebeck coefficient reverses around $40 \mathrm{~nm}$. The insets in (d) show schematic band structures and grey regions indicate the diameter range dominated by Bi surface states.

influence the bulk characteristics owing to their large surface/ volume ratio and SMSC transition. In the Bi NWs along the [100] direction, the critical diameter was found to be less than $40 \mathrm{~nm}$. The two-dimensional transport properties caused by surface-states were also revealed by the study of quantum oscillations using a $33 \mathrm{~nm}$ diameter OFFON-grown Bi NW. ${ }^{21}$ For the 21 and $27 \mathrm{~nm}$ diameter Bi NWs, the carrier concentrations induced from metallic surface-states were estimated to be $9.5 \times$ $10^{18}$ and $7.4 \times 10^{18} \mathrm{~cm}^{-3}$, respectively. These are greater than the concentration in the bulk $\left(3.0 \times 10^{18} \mathrm{~cm}^{-3}\right)$ and produce metallic behavior. The carrier concentrations were obtained from the surface-state density of $\mathrm{Bi}$ reported in previous studies $\left(n_{\mathrm{s}}=5 \times 10^{12} \mathrm{~cm}^{-2}\right.$, measured by angle-resolved photoemission spectroscopy) based on the relation, $n=2 n_{\mathrm{s}} / r$, where $r$ is the NW radius. ${ }^{16,33}$

These transitions in transport behavior were also confirmed by the diameter dependence of resistivity at room temperature (Fig. 2b). Although the four-probe method was employed, the measured resistivity was sensitive to the contact between the NW and the electrodes. Therefore, the additional four-probe devices were used to determine the diameter dependence of the resistivity accurately. The resistivity of Bi NWs with a large diameter $(d \gg 100 \mathrm{~nm})$ was consistent with that of bulk Bi $\left(\sim 2 \times 10^{-4} \Omega \mathrm{cm}\right)$ and the diameter dependence was negligible, which confirms the quality of the single-crystalline Bi NW. However, with the further decrease in diameter, the resistivity increased sharply owing mainly to the decreased mobility. The carrier non-specular surface scattering caused by the spatial constraints of NWs decreases the MFP. Moreover, the increase in the carrier effective mass caused by the strong coupling between electrons and holes also leads to a reduction of the mobility, as predicted theoretically ${ }^{4,8}$ and demonstrated experimentally $^{22}$ by SdH oscillations in a $100 \mathrm{~nm}$ diameter Bi NW. The significant increase of resistivity for the NWs with the diameter range of $40<d<110 \mathrm{~nm}$ could be attributed to the strong coupling effect, because the energy shift in the L-point sub-bands due to QSE dominates the spatial constraints of the Bi NWs in this diameter range. ${ }^{31}$ Furthermore, the SdH study reported a decrease in carrier concentration as a result of a change in the band overlap energy due to QSE, which also leads to an increase in resistivity. ${ }^{22}$ Finally, for the diameters below $30 \mathrm{~nm}$, the carrier concentration induced by the $\mathrm{Bi}$ surface states reduced the resistivity significantly, as mentioned above. However, the values of reduced resistivity could be compared with that of the large Bi NWs showing bulk resistivity characteristics owing to the high effective mass of the surface carriers. ${ }^{14}$ The variations in the measured resistances as well as the resistivities with temperature and diameter were in good agreement with the QSE and the surface-state effect of low-dimensional Bi (See ESI $\uparrow$ for detail).

\section{Seebeck coefficient}

Fig. 2c shows the temperature dependence of the Seebeck coefficient in the same samples as Fig. 2a. Physically, the Seebeck coefficient represents the entropy carried per unit charge; thus, the measured values converge to zero as the absolute temperature decreases. ${ }^{28}$ In a two-band system, the total Seebeck coefficient is determined by the partial Seebeck coefficients of each band weighted by their partial conduc- 
tivity. Semimetallic $\mathrm{Bi}$ has indirect conduction and valence bands, therefore the total Seebeck coefficient is given by ${ }^{1}$

$$
S_{\text {total }}=\frac{\sigma_{\mathrm{h}} S_{\mathrm{h}}+\sigma_{\mathrm{e}} S_{\mathrm{e}}}{\sigma_{\mathrm{h}}+\sigma_{\mathrm{e}}}
$$

where the subscripts $\mathrm{h}$ and e refer to holes and electrons, respectively. Because the partial Seebeck coefficients of the Tpoint hole and the L-point electron bands have opposite signs, the total Seebeck coefficient is relatively small despite the large absolute values of the partial coefficients $\left(S_{\mathrm{h}} \sim 670 \mu \mathrm{V} \mathrm{K}^{-1}\right.$ and $S_{\mathrm{e}} \sim-490 \mu \mathrm{V} \mathrm{K}^{-1}$ at $\left.300 \mathrm{~K}\right) .^{1,3,9,34}$ Consequently, the total Seebeck coefficient is extremely sensitive to intrinsic properties, such as carrier concentrations, carrier effective masses, and the MFP, which define the partial conductivities, i.e., the weighting factors in eqn (1). ${ }^{9,35}$ As shown in Fig. $2 \mathrm{~d}$, the decrease in the Seebeck coefficient as the diameter decreases to $40 \mathrm{~nm}$ can also be explained by a similar mechanism, involving the SMSC transition. The decrease in the band-overlap energy due to QSE with decreasing diameter can be determined from the confinement energy: ${ }^{4}$

$$
\Delta \varepsilon \sim \frac{\pi^{2} \hbar^{2}}{2 m_{\mathrm{c}}{ }^{*} d^{2}},
$$

where $\hbar$ and $m_{\mathrm{c}}{ }^{*}$ are the reduced Planck's constant and the carrier cyclotron effective mass, respectively. When the confinement energy is greater than the band-overlap energy (38-98 meV at 77-300 K), ,8,36 the SMSC transition occurs and produces a band gap. Previous theoretical studies have predicted that a change in band-overlap energy appears for diameters below $200 \mathrm{~nm}$ and leads to the formation of the band gap at approximately $d=50 \mathrm{~nm}$ at room temperature. ${ }^{3,4}$ Moreover, the transition diameter was calculated to be approximately $35 \mathrm{~nm}$ when considering the increase in carrier effective mass due to the strong coupling effect, which originates from the increase in the band gap energy of each subband (L-point electron and T-point hole sub-bands). Since the cyclotron effective mass of L-point electrons $\left(\sim 0.018 \mathrm{~m}_{\mathrm{e}}\right)$ is ten times smaller than that of T-point holes $\left(\sim 0.19 \mathrm{~m}_{\mathrm{e}}\right)$ in the Bi NW along the [100] direction, ${ }^{4,22}$ the L-point conduction (electron) sub-band rises faster than the lowering of the T-point valence (hole) sub-band with decreasing diameter (see eqn (2)). ${ }^{5}$ Consequently, the increase in effective carrier mass affects the L-point electrons more than the T-point holes, which leads to a decrease in the weighting factor of the L-point-electron partial Seebeck coefficient. ${ }^{22}$ A recent study reported that the increased cyclotron effective mass of electrons is greater than three times the bulk value for a diameter of $100 \mathrm{~nm}^{22}$ As a result, the absolute value of the total Seebeck coefficient decreased with decreasing diameter down to $40 \mathrm{~nm}$ according to eqn (1).

For diameters below $40 \mathrm{~nm}$, the SMSC transition is expected to increase the Seebeck coefficient through the formation of band gap. Although the absolute value of the measured Seebeck coefficient increased with the decrease in diameter below $40 \mathrm{~nm}$, the increase was small compared to the theoretical predictions. In practice, the largest measured value $\left(\sim-80 \mu \mathrm{V} \mathrm{K}^{-1}\right)$ was comparable to the maximum Seebeck coefficient reported in bulk $\mathrm{Bi}^{37}$ This can be attributed to the significant increase in the electron effective mass and the contribution of metallic surface states to the total Seebeck coefficient, which were not considered in the theoretical predictions. As shown in Fig. 2d insets, the band-overlap energy decreased with a reduction of diameter, but the band shifting itself delayed the SMSC transition, resulting in a decrease in the transition diameter due to the increase in the electron effective mass. Moreover, below the transition diameter, the band gap formed was dominated by the metallic surface states of Bi. However, the Seebeck coefficient of the $\mathrm{Bi}$ surface states exceeded the value of the Bi NWs because of the formation of the band gap, which eliminated the contribution of holes in the T-point valence sub-band below the transition diameter of the Bi NWs.

\section{Thermal conductivity}

Fig. 3 displays the diameter dependence of the thermal conductivity of Bi NWs, measured using the suspended microdevice (inset) at room temperature, with five different diameters 40, 69, 203, 230, and $301 \mathrm{~nm}$. The previous thermal conductivity data, obtained using single-crystalline Bi NWs grown by same method $(\mathrm{OFFON})^{23}$ and AAO templates, ${ }^{38}$ confirm the reliability of the measurements. Unlike the electrical thermoelectric properties, the thermal conductivity decreased monotonically as the diameter decreased from 310 to $40 \mathrm{~nm}$ and all the measured values in the Bi NWs were below that of bulk $\mathrm{Bi}^{39}$ The reduction of the thermal conductivity obtained in OFFON-grown Bi NWs, consistent with previous reports, can be attributed to the increase in diffuse phonon-surface scattering and partially diffuse surface scattering of carriers due to the classical size effect. ${ }^{38}$ The fact that the slope of the thermal conductivity with respect to the dia-

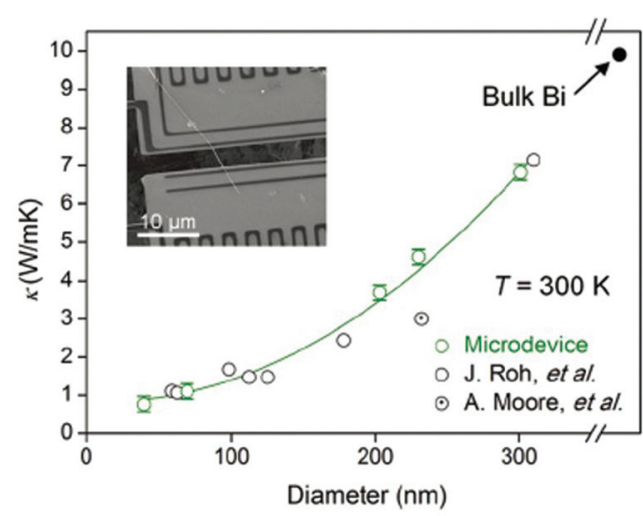

Fig. 3 Diameter-dependent thermal conductivity of single-crystalline Bi NWs: The thermal conductivity, measured using a suspended microdevice (inset), is plotted as a function of the diameter (green circles). The values measured by Roh et al. and Moore et al. were taken from ref. 23 and 25, respectively (black circles). Thermal conductivities were determined at the same diameters with power factor, by extrapolation (green curve). 
meter decreased with decreasing diameter signifies that phonon transport was sufficiently suppressed at a diameter of approximately $100 \mathrm{~nm}$. Thermal conductivity could not be obtained for Bi NWs with $d<40 \mathrm{~nm}$ because of the challenge of fabricating a suspended microdevice in this diameter range. A Bi NW with such a small diameter $(d<40 \mathrm{~nm})$ could not sustain when placed between the two membranes of the suspended microdevice, and a few dispersed Bi NWs were cracked during the drop casting. To place a selected Bi NW of such a diameter on the suspended microdevice, a nano-manipulator system $^{40,41}$ is preferred instead of the drop-casting method, in which the membranes were bent by the dropped solution.

\section{Power factor and figure of merit}

The power factor, which estimates electrical thermoelectric performance, is presented in Fig. 4a. It decreases from its value in bulk Bi to near zero at a $40 \mathrm{~nm}$ diameter, before increasing upon the further decreasing of the diameter. As mentioned in the description of the resistivity and Seebeck coefficient, reducing the NW diameter causes a variation of intrinsic Bi NW properties, which in turn decreases the power factor. Although the carriers induced by the metallic surface states of Bi increased the power factor for the NWs with diameters below $30 \mathrm{~nm}$, the enhancement was smaller than the
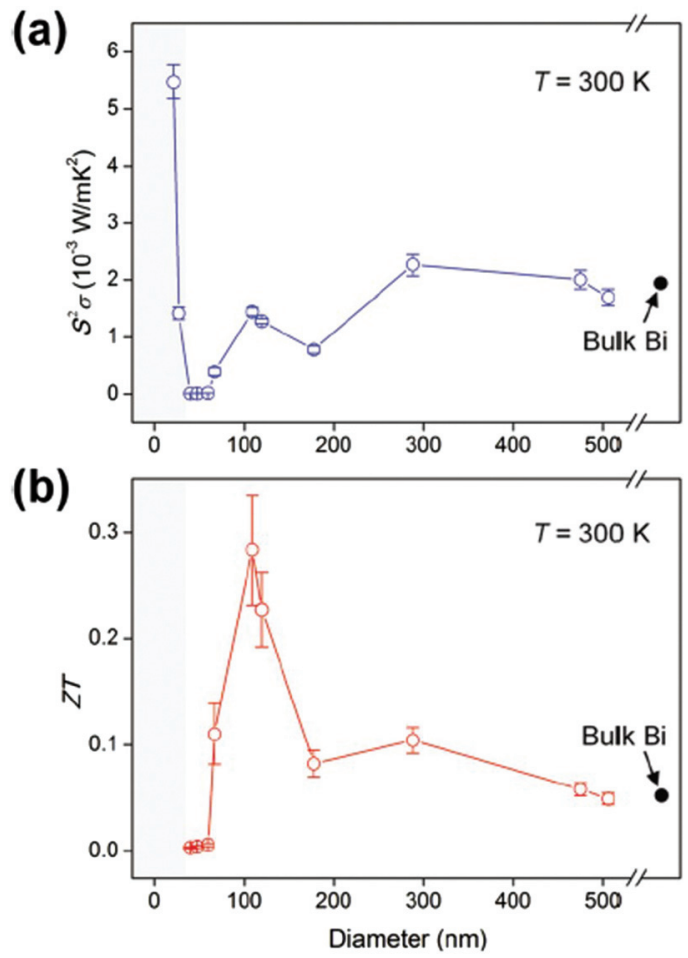

Fig. 4 Diameter-dependent power factor and $Z T$ of single-crystalline $\mathrm{Bi}$ NWs: (a) As the diameter decreases, the power factor decreases from its bulk value, except where surface states are dominant (grey area). (b) $Z T$ calculated from measured values increases with decreasing diameter, and decreases sharply after the peak at the diameter of $109 \mathrm{~nm}$. The large error in the peak value is mostly due to the error in the extrapolated thermal conductivity. theoretical prediction. Previous theoretical studies assumed that the SMSC transition in Bi leads to a significant increase of the Seebeck coefficient by reducing the two-band effect, which is explained in the Seebeck coefficient section. ${ }^{3,4}$ However, because of the increase of electron effective mass due to the strong coupling effect and the unexpected metallic surface states, the predicted effect is decreased by reducing transition diameter and overlapping the band gap. The enhancement of the Seebeck coefficient could not exceed the largest bulk value. $^{37}$

Finally, we calculated the figure of merit for single-crystalline Bi NWs as a function of diameter at room temperature using the measured thermoelectric properties. Because, the thermal conductivity of Bi NWs with $d<40 \mathrm{~nm}$ could not be obtained, $Z T$ values are given for $40 \mathrm{~nm}<d<500 \mathrm{~nm}$. As the diameter decreases within this range, whereas the thermal conductivity contributes to an increase in $Z T$, the variation of the power factor tends to decrease $Z T$. As a result, $Z T$ shows non-monotonic behavior with respect to the diameter, with a maximum value 0.28 for $d=109 \mathrm{~nm}$. This maximum is five times greater than the value in bulk Bi, as shown in Fig. $4 \mathrm{~b}$. It is noted that the enhancement of $Z T$ originates from the reduction of thermal conductivity, whereas the variation of the power factor has a negative contribution for diameters of 40-500 $\mathrm{nm}$. Moreover, despite the increase in the power factor for Bi NWs with a diameter less than $40 \mathrm{~nm}$, it is difficult to expect the $Z T$ enhancement, because the thermal conductivity can also be increased owing to the carrier density increase, according to Wiedemann-Franz law.

\section{Conclusions}

In summary, we have measured the diameter-dependent thermoelectric properties of single-crystalline Bi NWs grown by OFFON. The electrical resistivity, Seebeck coefficient, and thermal conductivity of Bi NWs were measured as functions of the diameter using TE devices and suspended microdevices based on a single NW. In contrast to theoretical predictions, as the diameter decreases to $40 \mathrm{~nm}$, the observed variations of resistivity and Seebeck coefficient, arising from the classical and quantum size effects, caused a decrease in the power factor. Although, when the diameter decreased below $30 \mathrm{~nm}$, the power factor started to increase because of $\mathrm{Bi}$ surface states, this increase was smaller than expected. Moreover, the thermal conductivity showed a monotonic dependency with diameter and contributed positively to $Z T$ in the measured diameter range. As a result, the calculated $Z T$ showed an increase relative to the bulk value, reaching a maximum value of 0.28 at a diameter of $109 \mathrm{~nm}$, followed by a decrease upon further decreasing the diameter. In low-dimensional Bi, enhancing $Z T$ is achieved more effectively via the suppression of the thermal conductivity than by controlling electrical thermoelectric properties, which make competing positive and negative contributions. We believe our findings not only provide an explanation for the thermoelectric properties of $\mathrm{Bi}$ NWs 
reported in previous studies, but also demonstrate the function of low-dimensional effects in thermoelectric materials. They therefore provide a basis for enhancing ZT using lowdimensional materials in the future.

\section{Acknowledgements}

This study was supported by the National Research Foundation of Korea (NRF) grant funded by the Korea government (MSIP) (2014R1A2A1A10053869), and the Priority Research Centers Program (2009-0093823) and the Pioneer Research Center Program (2013008070) through the NRF, and the Agency for Defense Development of Korea (ADD), under agreement number ADD-10-70-07-03.

\section{Notes and references}

1 L. D. Hicks and M. S. Dresselhaus, Phys. Rev. B: Condens. Matter, 1993, 47, 12727-12731.

2 L. D. Hicks and M. S. Dresselhaus, Phys. Rev. B: Condens. Matter, 1993, 47, 16631-16634.

3 M. S. Dresselhaus, G. Dresselhaus, X. Sun, Z. Zhang, S. B. Cronin and T. Koga, Phys. Solid State, 1999, 41, 679682.

4 Y. M. Lin, X. Z. Sun and M. S. Dresselhaus, Phys. Rev. B: Condens. Matter, 2000, 62, 4610-4623.

5 Z. B. Zhang, X. Z. Sun, M. S. Dresselhaus, J. Y. Ying and J. Heremans, Phys. Rev. B: Condens. Matter, 2000, 61, 48504861.

6 S. B. Cronin, Ph. D thesis, MIT, 2002.

7 J. Heremans, C. M. Thrush, Y. M. Lin, S. Cronin, Z. Zhang, M. S. Dresselhaus and J. F. Mansfield, Phys. Rev. B: Condens. Matter, 2000, 61, 2921-2930.

8 T. W. Cornelius and M. E. T. Molares, Nanowires, InTech, Shanghai, 2010.

9 J. Heremans and C. M. Thrush, Phys. Rev. B: Condens. Matter, 1999, 59, 12579-12583.

10 O. Rabin, Y. M. Lin and M. S. Dresselhaus, Appl. Phys. Lett., 2001, 79, 81-83.

11 Y.-M. Lin, O. Rabin, S. B. Cronin, J. Y. Ying and M. S. Dresselhaus, Appl. Phys. Lett., 2002, 81, 2403-2405.

12 T. E. Huber, A. Nikolaeva, D. Gitsu, L. Konopko, C. A. Foss and M. J. Graf, Appl. Phys. Lett., 2004, 84, 1326-1328.

13 T. E. Huber, A. Nikolaeva, L. Konopko and M. J. Graf, Phys. Rev. B: Condens. Matter, 2009, 79, 201304(R).

14 T. E. Huber, A. Adeyeye, A. Nikolaeva, L. Konopko, R. C. Johnson and M. J. Graf, Phys. Rev. B: Condens. Matter, 2011, 83, 235414.

15 A. Nikolaeva, T. E. Huber, D. Gitsu and L. Konopko, Phys. Rev. B: Condens. Matter, 2008, 77, 035422.

16 A. Nikolaeva, D. Gitsu, L. Konopko, M. J. Graf and T. E. Huber, Phys. Rev. B: Condens. Matter, 2008, 77, 075332.
17 E. Shapira, A. Holtzman, D. Marchak and Y. Selzer, Nano Lett., 2012, 12, 808-812.

18 W. Shim, J. Ham, K. I. Lee, W. Y. Jeung, M. Johnson and W. Lee, Nano Lett., 2009, 9, 18-22.

19 W. Shim, J. Ham, J. Kim and W. Lee, Appl. Phys. Lett., 2009, 95, 232107.

20 K. Lee, S. Lee, S. N. Holmes, J. Ham, W. Lee and C. H. W. Barnes, Phys. Rev. B: Condens. Matter, 2010, 82, 245310.

21 J. Kim, S. Lee, Y. M. Brovman, M. Kim, P. Kim and W. Lee, Appl. Phys. Lett., 2014, 104, 043105.

22 J. Kim, D. Kim, T. Chang and W. Lee, Appl. Phys. Lett., 2014, 105, 123107.

23 J. W. Roh, K. Hippalgaonkar, J. H. Ham, R. K. Chen, M. Z. Li, P. Ercius, A. Majumdar, W. Kim and W. Lee, ACS Nano, 2011, 5, 3954-3960.

24 S. Lee, J. Ham, K. Jeon, J.-S. Noh and W. Lee, Nanotechnology, 2010, 21, 405701.

25 A. L. Moore, M. T. Pettes, F. Zhou and L. Shi, J. Appl. Phys., 2009, 106, 034310.

26 A. I. Hochbaum, R. Chen, R. D. Delgado, W. Liang, E. C. Garnett, M. Najarian, A. Majumdar and P. Yang, Nature, 2008, 451, 163-167.

27 C.-H. Lee, G.-C. Yi, Y. M. Zuev and P. Kim, Appl. Phys. Lett., 2009, 94, 022106.

28 Y. M. Zuev, W. Chang and P. Kim, Phys. Rev. Lett., 2009, 102, 096807.

29 Y. M. Zuev, J. S. Lee, C. m. Galloy, H. Park and P. Kim, Nano Lett., 2010, 10, 3037-3040.

30 C. S. B. D. Schiferl, J. Appl. Crystallogr., 1969, 2, 30-36.

31 P. Hofmann, Prog. Surf. Sci., 2006, 81, 191-245.

32 T. Hirahara, K. Miyamoto, I. Matsuda, T. Kadono, A. Kimura, T. Nagao, G. Bihlmayer, E. V. Chulkov, S. Qiao, K. Shimada, H. Namatame, M. Taniguchi and S. Hasegawa, Phys. Rev. B: Condens. Matter, 2007, 76, 153305.

33 C. Ast and H. Höchst, Phys. Rev. Lett., 2001, 87, 177602.

34 J. Heremans and O. P. Hansen, J. Phys. Chem., 1979, 12, 3483-3496.

35 J. P. Heremans, C. M. Thrush, D. T. Morelli and M.-C. Wu, Phys. Rev. Lett., 2002, 88, 216801.

36 A. J. Levin, M. R. Black and M. S. Dresselhaus, Phys. Rev. B: Condens. Matter, 2009, 79, 165117.

37 B. S. Chandrasekhar, J. Phys. Chem. Solids, 1959, 11, 268273.

38 A. L. Moore, M. T. Pettes, F. Zhou and L. Shi, J. Appl. Phys., 2009, 106, 034310-034317.

39 C. F. Gallo, B. S. Chandrasekhar and P. H. Sutter, J. Appl. Phys., 1963, 34, 144-152.

40 K. Flöhr, M. Liebmann, K. Sladek, H. Y. Günel, R. Frielinghaus, F. Haas, C. Meyer, H. Hardtdegen, T. Schäpers, D. Grützmacher and M. Morgenstern, Rev. Sci. Instrum., 2011, 82, 113705.

41 S. Wang, Microsc. Microanal., 2006, 12, 1316-1317. 